\title{
SIM Grid Star Observations: Astrometry with a New High Dynamic Range Imaging Device
}

\author{
L. Winter ${ }^{1}$ \\ U.S. Naval Observatory (AD), Washington, DC 20392
}

\begin{abstract}
First astrometric and photometric applications of the logarithmically scaled HDRC4 imager (developed by the Institut für Mikroelektronik Stuttgart, IMS, Germany) are presented. To test the HDRC4 imager at the telescope, a cooled camera had to be constructed by modifying a camera designed by IMS. First observations were performed with various telescopes at Hamburg Observatory, Germany. These showed the dynamic range to exceed $14 \mathrm{mag}$ and the sensitivity to be comparable to that of the Kodak KAF $4200 \mathrm{CCD}$ at a chip temperature of $-35^{\circ} \mathrm{C}$.
\end{abstract}

\section{Introduction}

The HDRC4 (High Dynamic Range Camera) detector was developed by the Institut für Mikroelektronik Stuttgart (IMS, Germany) for high dynamic range applications, Seger et al. (1993), and Graf et al. (1995). It uses a logarithmically scaled sensitivity optimized for the visual bandpass as shown in Figure 1.

The characteristic curve of the HDRC4 (Figure 1, top) seems to be similar to that of a photographic plate. By changing the temperature of the imager, the sensitivity can be enhanced, which makes it possible to use the HDRC4 at the telescope. The sensitivity of the detector is wavelength dependent (Figure 1, bottom) with its maximum in the visual part of the spectrum. The response to blue light is comparable to a backside illuminated CCD. Due to the construction of the HDRC4 the light sensitive part of each pixel is not obstructed by a gate structure, thus no light is lost and no thining of the detector is needed.

The HDRC4 detector is in fact a matrix of photoelectric diodes arranged in a grid pattern. Each photoelectric diode has its own amplifier and switching transistor. The readout of each pixel is done by addressing the pixel, thereby activating the switching transistor, and measuring the output voltage of the amplifier. Doing so will measure the current flowing through the photodiode. Therefore the HDRC4 measures dynamically and not statically like a CCD or a photographic plate. Cooling the HDRC4 results in a reduction of the dark current flowing through the photodiode and decreasing the leakage currents as well. This increases the mean storage time of the electrons, which is somewhat similar to increasing the exposure time on any other camera.

\footnotetext{
${ }^{1}$ also with Universities Space Research Association (USRA), Washington, DC
} 
After testing a HDRC4 camera on loan from IMS successfully in January 1998 by taking pictures of bright objects like the moon and some planets with a telescope, a thermoelectrically cooled camera was constructed to estimate the maximum usable magnitude range and the limiting magnitude of the device.

\section{Observations obtained at Hamburg Observatory}

The open cluster h Persei was choosen to determine the properties of the cooled HDRC4 detector and compare them to a Kodak CCD KAF4200. The telescope used was the large refractor $(f=9 \mathrm{~m}, \mathrm{D}=0.60 \mathrm{~m})$ at Hamburg Observatory in November, 1998, and January, 1999, under good sky conditions with a seeing FWHM of $\approx 2$ arcsec.

Figure 2 (left) demonstrates the influence of detector temperature on the limiting magnitude: a single picture taken at $T_{\text {sens }}=+1^{\circ} \mathrm{C}$ (top) shows a very limited number of stars as compared to the picture taken at $T_{\text {sens }}=-35^{\circ} \mathrm{C}$ (bottom). The gain visible in Figure 2 (left) is a result of the increased integration time, as described above.

Figure 2 (right) is a comparison of the processed HDRC4 image having an equivalent exposure time of $100 \mathrm{sec}$ and a CCD camera frame with an exposure time of $100 \mathrm{sec}$. The images were taken on two different nights with nearly identical sky conditions. Both pictures show the same stars, but the brightest star cannot be measured on the CCD frame, because it is already overexposed. On the HDRC4 detector the star can be measured with a very high accuracy (Figure 5).

Figure 3 demonstrates the dynamic range of the CCD camera (left) and that of the HDRC4 (right). Each picture shows a bright star and its influence on the detector. The CCD shows the well known overexposure effects: charge is bleeding along the columns and the amplifier cannot handle the fast transition in intensity, resulting in a shadow. The HDRC4 image depicts Deneb (V=1.25 $\mathrm{mag}$ ). The faintest stars shown on the original picture are of magnitude 15.5. A grating was used in front of the telescope (zone astrograph: $f=2 \mathrm{~m}, \mathrm{D}=0.23$ $\mathrm{m}, 6^{m}$ grating) to estimate the limiting magnitude. This grating produces side images (first and higher order spectra of Deneb), which are not overexposure effects.

\section{Results}

It can be demonstrated by the measurements in $h$ Persei that the response of the HDRC4 detector to light is logarithmic for stars of magnitude 11 and brighter (Figure 4, left). The faint stars show an almost linear response in the magnitude range 12 to 14 . This is due to the knee of the characteristic curve (Figure 1, left). The astrometry of all the images taken with the HDRC4 so far is compatible to a CCD except that the dynamic range of the HDRC4 exceeds that of any other detector. It almost doubles the usable delta mag (Figure 5, left). 

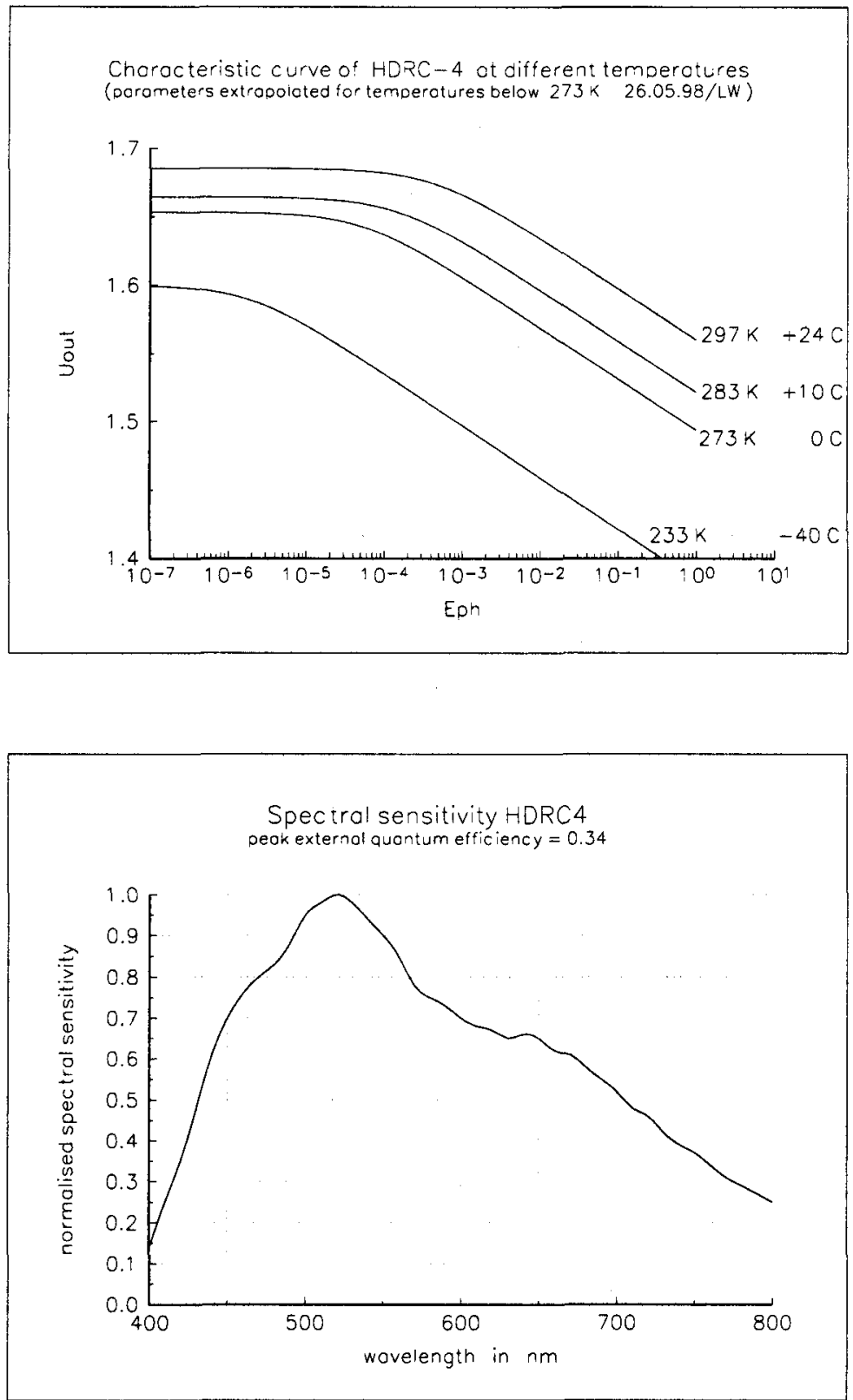

Figure 1. Characteristic curve and spectral sensitivity of the HDRC4. 

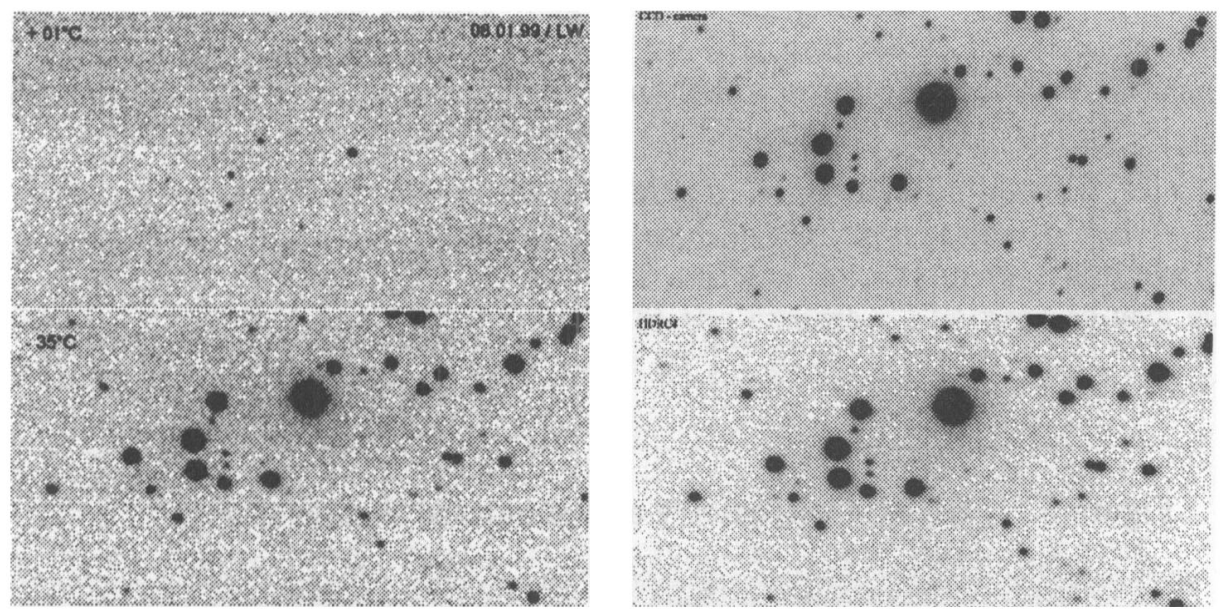

Figure 2. Characterizing the HDRC4 imager: the left two images show the temperature dependent integration time of the HDRC4. On the right hand side the processed image of a KAF4200 CCD (top) is compared to that of the HDRC4 (bottom).
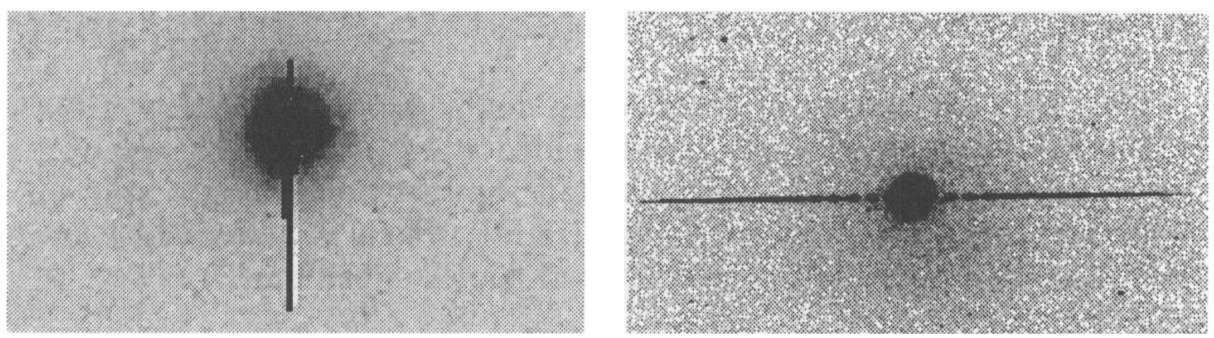

Figure 3. Overexposed star image of a $V=7.5$ mag star on a Kodak KAF4200 CCD (left hand side) and the star field around the bright star Deneb (V=1.25 mag) on HDRC4 (right hand side) with a limiting magnitude of $15.5 \mathrm{mag}\left(6^{m}\right.$ grating used).
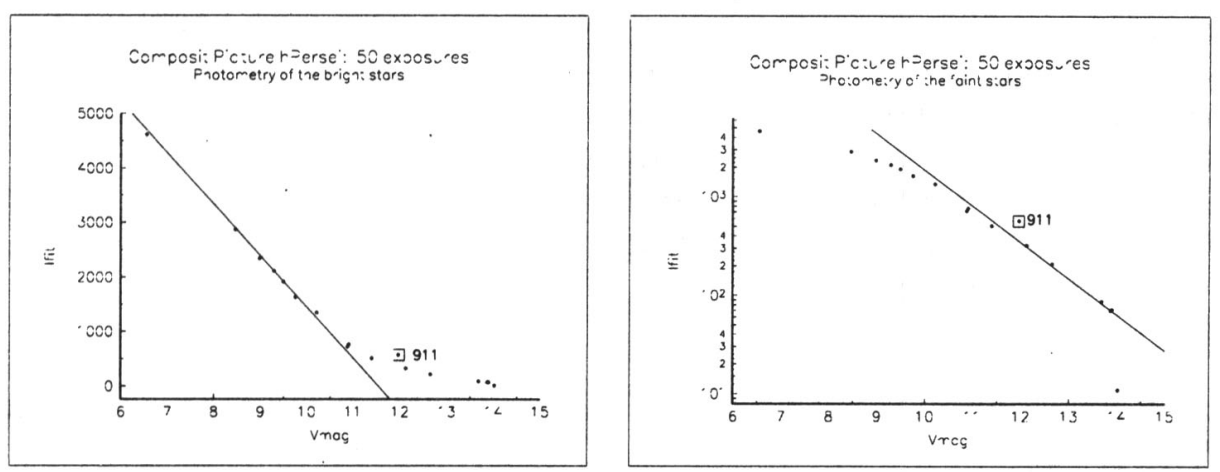

Figure 4. HDRC4 photometry for bright and faint stars in $\mathrm{h}$ Persei. 

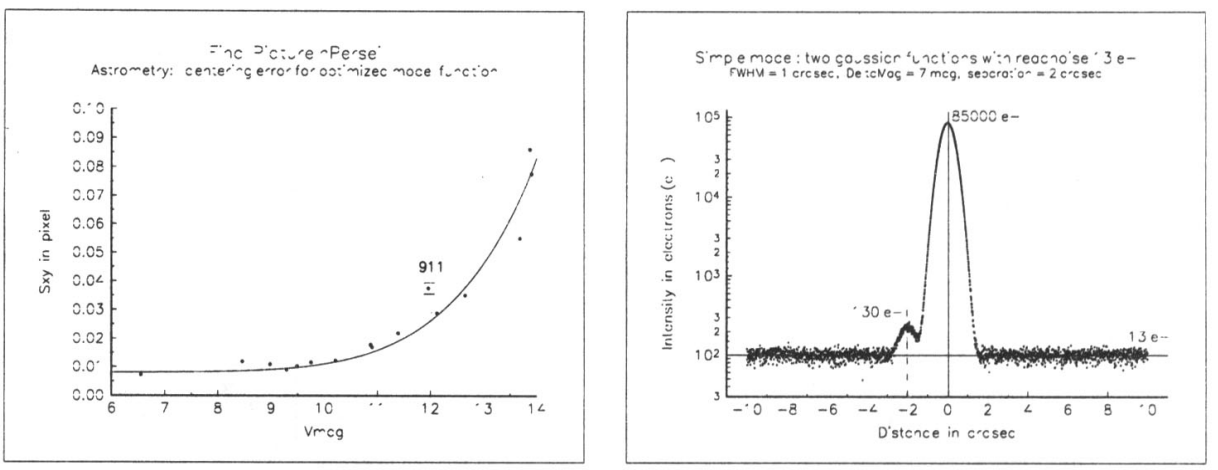

Figure 5. HDRC4 astrometry and simulated double star.
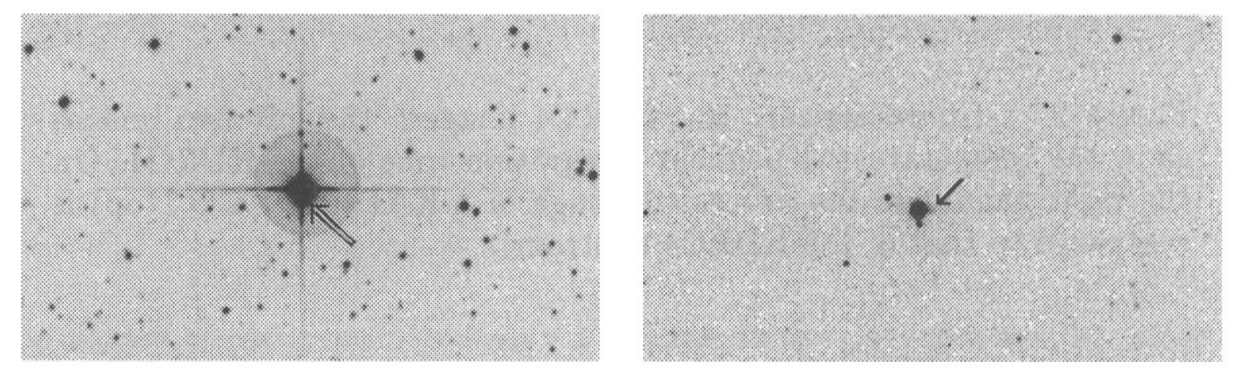

Figure 6. Difficulties in detectecting double stars: The spikes of the Schmidt telescope will mask faint double stars (left, DSS image of a close double star). The comet like tail shown by each bright star on this USNO CCD astrograph image (right), which is caused by a low CTE, poses problems for astrometry of faint companions.

\section{Detection of double stars}

As part of the USNO grid star selection for SIM, direct photography of double stars with a separtion of more than two arcseconds at a delta mag of up to 7 was undertaken. The telescopes and detectors used have to be choosen with great care, as demonstrated in Figure 6. Any telescope with spiders in the optical path will produce diffraction spikes in the image, as can be seen in this DSS image (Figure 6 , left). The double star companion (indicated by the arrow) is almost lost in the defraction pattern. Faint double stars can be mimicked by the diffraction spikes or by the comet-like tail, which is a result of a low CTE, as shown on the USNO CCD astograph image (Figure 6, right). To detect double star companions with a high delta mag is not as easy as the simulation (Figure 5 , right) seems to suggest.

\section{Conclusions}

Due to the high dynamic range of the HDRC4 sensor and its logarithmically scaled sensitivity it is a natural choice to detect close double stars with large magnitude differences. Simulations show that the HDRC4 camera is able to 
detect double stars with a delta mag of 7 at a separation of $2 \operatorname{arcsec}$ (seeing FWHM 1 arcsec), as is needed to evaluate the SIM grid stars. More observations are scheduled at Lick Observatory in May, 2000, using an improved version of the cooled HDRC4 camera and the same CCD camera.

Acknowledgments. We thank the IMS, U. Seger, B. Höfflinger, R. Glatthaar, for providing a prototype HDRC4 camera and helpful discussions to understand the working of the HDRC4. C. de Vegt and the workshop in Hamburg are thanked for building the cooled version of the camera. The project was supported by BMBF (AMECC-Studie, FKZ: 50 TT 9740).

\section{References}

Graf, H.E., Höfflinger, B., Seger, U., Siggelkow A., 1995, Electronik 3/95.

Seger, U., Graf, H.E., \& Landgraf, M.E., 1993, IEEE Micro, 50. 